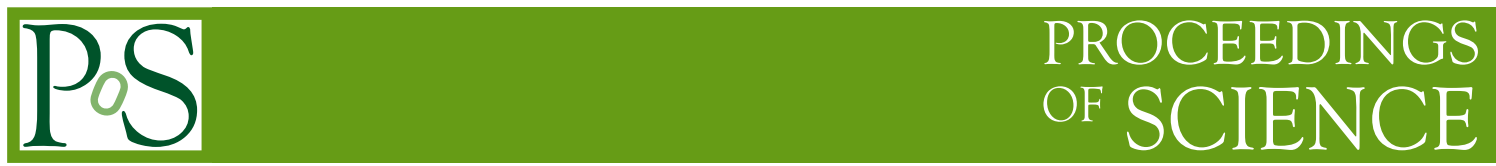

\title{
Exclusive diffractive Higgs and jet production at the LHC
}

\section{Christophe Royon*}

IRFU-SPP, CEA Saclay, F91 191 Gif-sur-Yvette cedex, France

E-mail: christophe.royon@cea.fr

\section{Rafal Staszewski}

CEA/IRFU/Service de physique des particules, CEA/Saclay

Institute of Nuclear Physics, Polish Academy of Sciences, Krakow

E-mail: rafal.staszewskilifj.edu.pl

\section{Alice Dechambre}

IFPA, Dept. AGO, Université de Liège

E-mail: alice.dechambre@ulg.ac.be

\section{Oldrich Kepka}

Center for Particle Physics, Institute of Physics, Academy of Science, Prague

E-mail: kepkao@fzu.cz

We describe the implementation of exclusive diffractive production of Higgs boson and dijets in the Forward Physics Monte Carlo following the models by Khoze, Martin, Ryskin (KMR) and Cudell, Dechambre, Hernandez and Ivanov (CHIDe). The predictions of the models are compared to the CDF measurement of exclusive jets and the uncertainties on the Higgs boson and jet production cross sections at the LHC are discussed.

35th International Conference of High Energy Physics - ICHEP2010,

July 22-28, 2010

Paris France

\footnotetext{
${ }^{*}$ Speaker.
} 
We discuss in these proceedings the implementation of the exclusive diffractive jet and Higgs boson production in the Forward Physics Monte Carlo (FPMC). Both incoming hadrons, $p \bar{p}$ at the Tevatron and $p p$ at the LHC, remain intact after the interaction and the Higgs decays in the central region. The process involves the exchange of a color singlet, thus large rapidity gaps can remain between the Higgs boson and the outgoing hadrons. The great advantage of such a production mechanism is the possibility to detect fully exclusive events by tagging both outgoing hadrons As an example, a mass resolution on the Higgs boson of 2 to $3 \mathrm{GeV}$ according to the domain in mass can be achieved using such events.

\section{Theoretical models implemented in FPMC}

The exclusive production can be modeled within QCD. In the simplest case the process can be described as a two-gluon exchange - one gluon involved in the production and the other one screening the color. The theoretical calculation is model dependant because of the following factors: impact factor, Sudakov form factor and rapidity gap survival probability.

The impact factor [1] regulates the infra-red divergence and embeds quarks inside the proton. It is modeled phenomenologicaly and includes soft physics. The Sudakov form factor [2] corresponds to virtual vertex corrections and depends on two scales - the hard scale linked to the hard subprocess $(g g \rightarrow X)$ and the soft scale related to the transverse momentum of the active gluons - the scale from which a virtual parton can be emitted. The Sudakov form factor suppresses the cross section by a factor of the order of 100 to 1000 . Finally, additional soft interactions of initial and final state protons can occur [3], which are taken into account by introducing the rapidity gap survival probability.

In this work we study two models of exclusive Higgs and jets production: the Khoze, Martin and Ryskin (KMR) model [2, 4] and the Cudell, Hernández, Ivanov, Dechambre exclusive (CHIDe) model [5]. The models are in fact very similar - both use perturbative QCD calculations and have similar ingredients. However they differ in details, which leads to different predictions.

Three main differences are present between the KMR and CHIDe models. The first difference is the collinear approximation used in the KMR model contrary to the exact kinematics used in CHIDe. The second one is the variable used as the upper scale of the Sudakov form factor in the exclusive jet case. It is chosen as the gluon-gluon invariant mass, $s_{g g}$, in the KMR model, whereas in the CHIDe model the transverse momentum squared of the gluon, $k_{T}$, is used. The last difference is the impact factor in CHIDe model that suppresses very soft gluon emissions from the proton, which is not present in the KMR model.

\section{FPMC - Forward Physics Monte Carlo}

The Higgs and jet exclusive production in both KMR and CHIDe models have been implemented in the Forward Proton Monte Carlo (FPMC) [6], a generator that has been designed to study forward physics, especially at the LHC. It aims to provide a variety of diffractive processes in one common framework, i.e. single diffraction, double pomeron exchange, central exclusive production and two-photon exchange. 


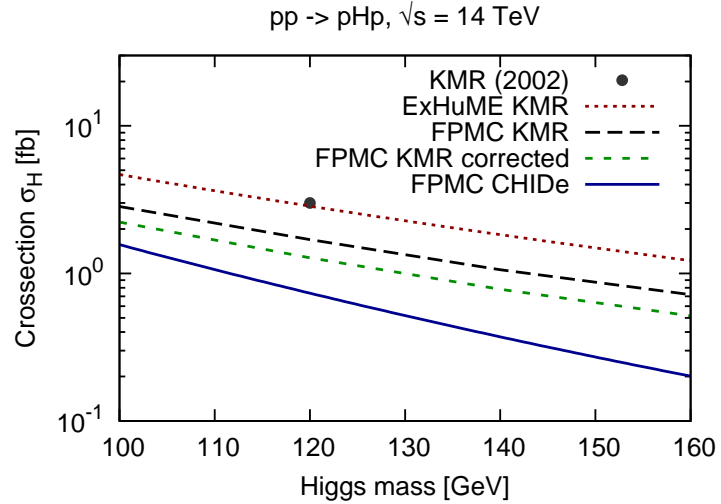

Figure 1: Cross section for exclusive Higgs production at the LHC for various models.

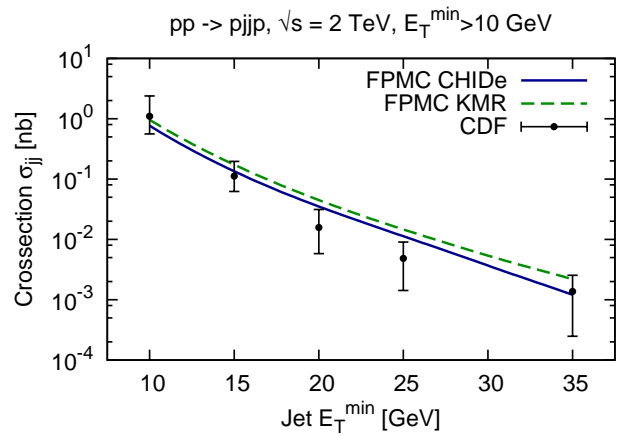

Figure 2: Exclusive jets production cross section at the Tevatron as a function of jets $E_{T}^{\min }$.

The implementation of the KMR and CHIDe models in FPMC allows their direct comparison using the same framework. In Fig. 1, we present the cross section of exclusive Higgs boson production at the LHC as a function of the Higgs boson mass. In addition, we show the predictions from the KMR original calculation [4] and the results of the implementation of the KMR model in the ExHuME generator [7]. The difference in the results between the FPMC and ExHuME implementations of the KMR model is mainly due to the way the gluon density is implemented. In ExHuME the value of the gluon distribution is frozen for small $Q^{2}$, whereas in FPMC it vanishes to 0 .

The predictions of the KMR and CHIDe models are compared to the CDF measurement of exclusive jets production at the Tevatron (Fig. 2). A good agreement is found between the CDF measurement and the predictions of both CHIDe and KMR models. The difference between the models is small compared to the data uncertainties.

\section{Model uncertainties}

In this section, we discuss the different uncertainties associated with the models of exclusive diffractive processes. We restrict our analysis to the CHIDe model, expecting the results for the KMR model to be qualitatively similar. There are three main sources of uncertainties: the gap survival probability which will be measured using the first LHC data (in this study we assume a value of 0.1 at the Tevatron and 0.03 at the LHC [8]), the gluon density, which contains the hard and the soft part (contrary to the hard part, the soft one is not known precisely and originates from a phenomenological parametrisation), and finally the limits of the Sudakov integral, which have not yet been fixed by theoretical calculations (apart from the upper limit for the Higgs case) and thus are not known precisely.

To check the uncertainty due to the gluon distributions four different parametrisations of unintegrated skewed gluon densities are used to compute the exclusive jet and Higgs boson cross sections. These four gluon densities represent the uncertainty spread due to the present knowledge of unintegrated parton distribution functions. All of them lead to a fair agreement with the Tevatron 


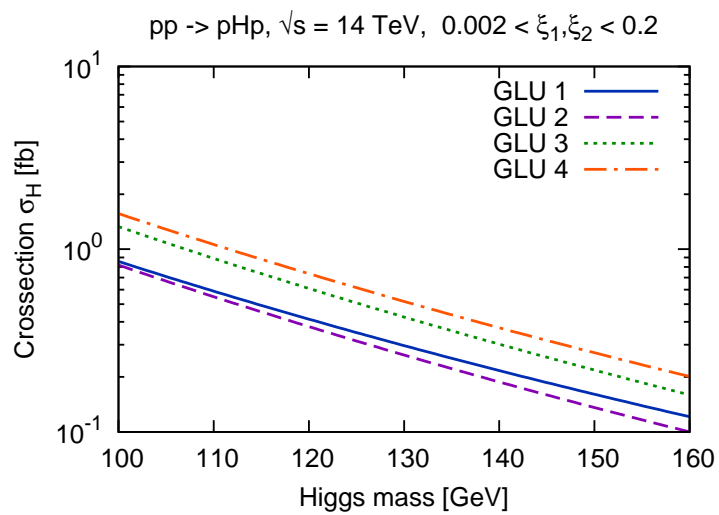

Figure 3: Uncertainty due to the gluon distributions for exclusive Higgs at the LHC.

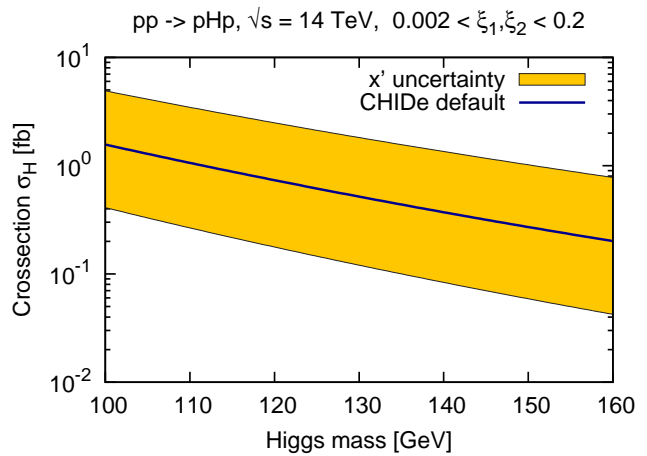

Figure 4: Uncertainty due to the lower Sudakov form factor limit for exclusive Higgs at the LHC.

exclusive jet measurement and they lead to an uncertainty of about a factor of 3.5 for jets and 2 for Higgs boson exclusive production at the LHC, The results for the Higgs boson production cross section are presented in Fig. 3.

To analyse the uncertainties coming from the Sudakov form factor, both upper and lower limits of the integral are varied by a factor 2 . The study shows that the effect of changing the upper scale is smaller than for the lower scale. This is especially true at LHC energies, where the upper scale uncertainty can be usually neglected. In Fig. 4 we show the uncertainty of the lower scale for Higgs boson production at the LHC.

\section{Predictions at the LHC}

After discussing the model uncertainties, it is important to study if they can be reduced using present Tevatron data, which will allow to make more precise predictions especially for Higgs boson production at the LHC. The basic idea is to consstrain the model uncertainties with the CDF measurement and use the obtained values at the LHC energy. We take into consideration both the gluon uncertainty and the dominant, lower limit of the Sudakov form factor calculation. The principle is simple: for each gluon density (GLU1 to GLU4), we choose a range of lower limit values which are compatible with the $\mathrm{CDF}$ measurement, taking into account the CDF data error. The same limit values are used at LHC energies to predict the jet (Fig. 5) and Higgs (Fig. 6) cross sections. The obtained uncertainty is large, the factor between the lower and upper edges of the uncertainty is larger than 10 for jets and about 25 for Higgs production.

In order to contrain further the uncertainty on the Higgs boson cross section, we study the possible constraints using early LHC measurements of exclusive jets using an integrated luminosity of $100 \mathrm{pb}^{-1}$. In addition to the statistical uncertainties, we consider a conservative $3 \%$ jet energy scale uncertainty as the dominant contribution to the systematic error. A possible result of such a measurement is presented in Fig. 5. Using the same prescription as before, we obtain the prediction for Higgs boson prediction as shown in Fig. 6. 


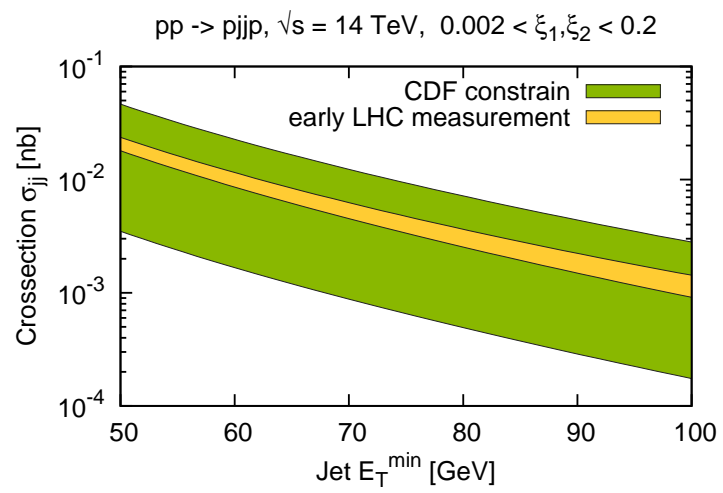

Figure 5: Total uncertainty for exclusive jets at the LHC: constraint provided by the CDF measurements and the potentail LHC measurement with a low luminosity of $100 \mathrm{pb}^{-1}$.

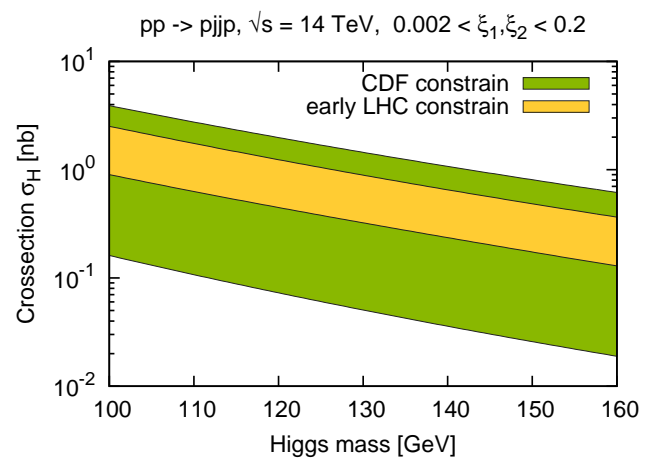

Figure 6: Total uncertainty for exclusive Higgs production at the LHC: constraint provided by the CDF measurements and the potentail LHC measurement with a low luminosity of $100 \mathrm{pb}^{-1}$.

Both KMR and CHIDe models describe fairly the CDF measurement of exclusive jets, but at LHC energy their predictions differ. Without taking into account the constraints from the CDF measurement, and possible LHC maesurements, the total uncertainty for exclusive production at the LHC is large. It is possible to constrain the Higgs boson cross sections within a factor 2 using early LHC measurement of exclusive jets. It is also useful to notice that additional models not described in these proceedings were also implemented in FPMC. As an example, extra dimension or higgsless midels can be probed via anomalous couplings [9] between $\gamma$ and $W$ or $Z$ bosons which have been implemented in FPMC.

\section{References}

[1] I. P. Ivanov, N. N. Nikolaev and A. A. Savin, Phys. Part. Nucl. 37 (2006) 1 [arXiv:hep-ph/0501034].

[2] V. A. Khoze, A. D. Martin and M. G. Ryskin, arXiv:hep-ph/0006005.

[3] L. Frankfurt, C. E. Hyde, M. Strikman and C. Weiss, Phys. Rev. D 75, 054009 (2007) [arXiv:hep-ph/0608271].

[4] V. A. Khoze, A. D. Martin and M. G. Ryskin, Eur. Phys. J. C 23, 311 (2002) [arXiv:hep-ph/0111078].

[5] J. R. Cudell, A. Dechambre, O. F. Hernandez and I. P. Ivanov, Eur. Phys. J. C 61 (2009) 369 [arXiv:0807.0600 [hep-ph]].

[6] M. Boonekamp, V. Juranek, O. Kepka, C. Royon "Forward Physics Monte Carlo", "Proceedings of the workshop: HERA and the LHC workshop series on the implications of HERA for LHC physics," arXiv:0903.3861 [hep-ph].

[7] J. Monk and A. Pilkington, Comput. Phys. Commun. 175, 232 (2006) [arXiv:hep-ph/0502077].

[8] V. A. Khoze, A. D. Martin and M. G. Ryskin, Eur. Phys. J. C 18, 167 (2000) [arXiv:hep-ph/0007359].

[9] O. Kepka and C. Royon, Phys. Rev. D 78 (2008) 073005; .E. Chapon, O. Kepka, C. Royon, Phys. Rev. D81 (2010) 074003; J. de. Favereau et al., preprint arXiv:0908.2020. 